\title{
Oleuropein is a Powerful Sensitizer of Doxorubicin-mediated Killing of Prostate Cancer Cells and Exerts Its Action via Induction of Autophagy
}

\author{
Anastasia Papachristodoulou ${ }^{1,2}$, Magafoula Tsoukala ${ }^{1,2}$, Dimitra Benaki ${ }^{2}$, Sarantos Kostidis ${ }^{2}$, Katerina Gioti ${ }^{1}$, \\ Nektarios Aligiannis ${ }^{4}$, Harris Pratsinis ${ }^{3}$, Dimitris Kletsas ${ }^{3}$, Alexios-Leandros Skaltsounis ${ }^{4}$, Emmanuel Mikros ${ }^{2, *}$, \\ Roxane Tenta ${ }^{1, *}$ \\ ${ }^{1}$ Department of Nutrition Science and Dietetics, Harokopio University, Athens, Greece \\ ${ }^{2}$ Division of Pharmaceutical Chemistry, Faculty of Pharmacy, National and Kapodistrian University of Athens, Greece \\ ${ }^{3}$ Laboratory of Cell Proliferation and Ageing, Institute of Biosciences \& Applications, National Centre for Scientific Research \\ "Demokritos", Athens, Greece \\ ${ }^{4}$ Division of Pharmacognosy and Chemistry of Natural Products, Faculty of Pharmacy, National and Kapodistrian University of Athens, \\ Greece \\ *Corresponding authors: rtenta@hua.gr, mikros@pharm.uoa.gr
}

\begin{abstract}
The phenolic component Oleuropein (OLEU), a bioactive natural product, has recently shown antiproliferative properties. Doxorubicin (DXR) is an anthracycline present in many chemotherapeutic schemes, although limited due to its cardio-toxic effects. Important research effort has been devoted therefore, to reducing DXR toxicity without compromising its antitumor efficacy. The anticancer actions of DXR and OLEU were assessed, on PC-3 prostate cancer cells, while cell cycle distribution and rate of apoptosis were assessed by flow cytometry. The autophagic process was determined via immunoblotting and immunofluorescent staining. Finally, cell extracts were analyzed by NMR spectroscopy. The present study showed that both DXR and OLEU inhibited PC-3 cells proliferation, while the co-treatment with DXR and OLEU resulted in an additive inhibition. Although the addition of OLEU to DXR did not alter significantly the cell cycle distribution, exhibited by each treatment alone, and produced a marginal increase on the rate of apoptosis, both compounds produced a remarkable induction of autophagy. In addition, treated cells exhibited significant metabolite alterations. This study demonstrates that OLEU, a basic component of the everyday diet, is capable of lowering significantly the cytotoxic dose of DXR, while obtaining an important anti-proliferative effect in prostate cancer cells.
\end{abstract}

Keywords: oleuropein, doxorubicin, autophagy, prostate, metabolic profiling

Cite This Article: Anastasia Papachristodoulou, Magafoula Tsoukala, Dimitra Benaki, Sarantos Kostidis, Katerina Gioti, Nektarios Aligiannis, Harris Pratsinis, Dimitris Kletsas, Alexios-Leandros Skaltsounis, Emmanuel Mikros, and Roxane Tenta, "Oleuropein is a Powerful Sensitizer of Doxorubicin-mediated Killing of Prostate Cancer Cells and Exerts Its Action via Induction of Autophagy." Journal of Cancer Research and Treatment, vol. 4, no. 4 (2016): 61-68. doi: 10.12691/jcrt-4-4-2.

\section{Introduction}

Prostate cancer is very common in Western populations and is the second leading cause for male death from cancer in North America. The incidence of cancer in Mediterranean countries is lower than in the rest of European countries and the United States. This is mostly described by the lower rate of the large bowel, breast, endometrial, and prostate cancers by a number of epidemiological studies, and the major reason for this, apart from possible genetic factors, is attributed to the dietary practices [1]. The traditional Mediterranean diet is characterized by high consumption of foods of plant origin, relatively low consumption of red meat, and high consumption of olive oil and its products [2].
Doxorubicin (DXR) is a potent anthracycline antibiotic that has been used in anticancer therapy for decades. However, DXR is also well known to exert toxic effects on normal tissues. Especially in the heart, DXR can induce an accumulative dose-dependent cardiomyopathy that ultimately leads to congestive heart failure. Despite its severe toxicity, DXR remains a major component of most chemotherapeutic regimens because of its efficacy and broad spectrum antitumor activity. As a result, sustained research effort has been devoted to identifying effective drugs or strategies that can reduce DXR toxicity without compromising its antitumor efficacy [3].

Since olive oil is one of the most valuable nutritional agents and is also the major energy source in the Mediterranean diet, several studies were recently conducted on the beneficial effects of olive oil (extracted mechanically from olives, the fruit of Olea europaea $L$ ) and on oleuropein, the most prominent phenolic 
compound in olive fruit and leaves. Oleuropein (OLEU) has several pharmacological properties, including antioxidant, anti-inflammatory, anti-atherogenic and anticancer actions and for these reasons, it is commercially available as food supplement in Mediterranean countries $[4,5]$. Although Oleuropein has been shown to exhibit anti-proliferative activity in vitro against a number of cancer types, the underlying action mechanism remains largely unknown [6,7]. In addition, OLEU has been shown to be cardioprotective against acute doxorubicin cardiotoxicity and has been shown to exhibit anti-ischemic and hypolipidemic activities [8,9].

The healthy effects of plant polyphenols, some of which characterize the so-called Mediterranean diet, have been shown to arise from epigenetic and biological modifications resulting, among others, in autophagy stimulation. Autophagy is one of the major pathways for degradation of cellular components in animal cells that controls the turnover of long-lived proteins and organelles. Although initially identified as a process induced by cellular starvation, an autophagic pathway is now recognized as the cellular response to a variety of stimuli $[10,11]$. During autophagy, cytoplasmic components are engulfed by double membrane-bound structures (autophagosomes) and delivered to lysosomes for degradation. During autophagosome formation, a cytosolic microtubuleassociated protein-light chain 3 (LC3) is processed and conjugated with phosphatidylethanolamine (PE). The PEconjugated LC3, LC3-II, is inserted into autophagic vesicle membranes. Because the amount of LC3-II correlates with the number of autophagosomes, LC3-II is widely considered as an autophagosomal marker for monitoring autophagy [12,13].

The objective of the present study was the evaluation of the cytostatic action of OLEU, DXR and, more interestingly, of the combined treatment with OLEU and DXR, on PC-3 prostate cancer cells. Furthermore, the scope of this study was the investigation of the mechanism of action of OLEU and DXR, in terms of the impact on the autophagic pathways and the identification of the metabolic profile of prostate cancer cells.

\section{Materials and Methods}

\subsection{Oleuropein Isolation}

The oleuropein used in this study was isolated from Olea europaea (Oleaceae) leaves as described before [8]. Briefly, air-dried and pulverized leaves were extracted with mechanical stirring for $12 \mathrm{~h}$ with acetone. The extract was evaporated to dryness and was washed with a mixture of dichloromethane/methanol (98/2). The insoluble material was separated, dried and submitted to medium pressure liquid chromatography with Si gel 60 Merck, using dichloromethane-methanol gradient as the eluent to afford pure oleuropein.

The structure elucidation of oleuropein was carried out using spectroscopic and spectrometric methods as well as comparison with literature data [14]. Specifically, 1D\&2D NMR spectra (COSY, COSYLR, HSQC-DEPT, HMBC) were recorded in deuterated methanol ( $\mathrm{CD}_{3} \mathrm{OD}$ - Merck), on a Buker Avance III spectrometer (Bruker Biospin $\mathrm{GmbH}$, Reinsteten, Germany) operating at $600.11 \mathrm{MHz}$ for $1 \mathrm{H}$ and at $150.11 \mathrm{MHz}$ for $13 \mathrm{C}$, with a $5 \mathrm{~mm}$ inverse detection probe. The residual $1 \mathrm{H}(3.33 \mathrm{ppm})$ and $13 \mathrm{C}$ (49.50 ppm) signals of $\mathrm{CD}_{3} \mathrm{OD}$ were used as internal standard. 1D \& 2D NMR experiments were performed with standard pulse programs, at room temperature. HRMS \& HRMS/MS data were obtained by direct infusion method using an hybrid LTQ-Orbitrap Discovery Mass Spectrometer (Thermo Scientific, Bremen, Germany) equipped with an ESI probe, in positive mode. Oleuropein's purity (95\%) is the maximum that can be obtained from a natural compound.

\subsection{Cell Line}

The PC-3 cells, an androgen insensitive, p53-negative and K-Ras mutated human prostate cancer cell line, were obtained from the American Type Cell Culture (ATCC, Bethesda, $\mathrm{Md}$ ). This cell line was grown in $75-\mathrm{cm}^{2}$ culture flasks at $37^{\circ} \mathrm{C}$ in $5 \% \mathrm{CO}_{2}$ using Dulbecco's modified Eagle's medium F/12 (DMEM/F-12, Gibco, USA) containing $10 \%$ fetal bovine serum (FBS, Gibco, USA) [15].

\subsection{MTT Proliferation Assay}

PC-3 cells were plated at a cell density of 500 cells/well in 96-well plates $24 \mathrm{hrs}$ prior to treatment with the appropriate drug at various concentrations for $96 \mathrm{hrs}$. The anticancer actions of DXR (3-100 nM) and of OLEU (3$400 \mu \mathrm{g} / \mathrm{mL}$ ) were assessed, alone and in co-treatment, on PC-3 cells. The cells were incubated with 10\% MTT (Sigma M-5655, USA) added directly in the medium for 4 hrs at $37^{\circ} \mathrm{C}$. The medium was then aspirated and the cells were solubilized with the organic solvent dimethylsulfoxide (DMSO). Absorbance was determined in a VERSA max microplate reader (Molecular Devices Corporation, USA) at $540 \mathrm{~nm}$ and results are presented as the percent of OD in the treated wells versus the controls [16].

\subsection{Flow Cytometry}

Cell-cycle analysis was performed following incubation of PC-3 cells with DXR (50 nM) and OLEU $(200 \mu \mathrm{g} / \mathrm{mL})$, alone and in co-treatment, for 24, 48 and 72 hours. Cells were then washed in PBS, fixed in 50\% ethanol solution (v/v), and stained with an RNAse-containing propidium iodide (PI) solution $(50 \mu \mathrm{g} / \mathrm{ml})$. DNA content was analyzed on a FACS Calibur (Becton Dickinson, San Jose, CA) flow cytometer using the ModFit software (Verity Software House, Topsham, ME). Apoptosis of PC-3 cells was assessed by analysing DNA content in Annexin VFITC and propidium iodide (PI) stained cells (Annexin VFITC Apoptosis Detection Kit, BD Systems) on a BD Accuri C6 flow cytometer.

\subsection{Immunofluorescence Labeling}

For the detection of the autophagic marker LC-3, PC-3 cells were plated at a cell density of 20,000 cells / well on Lab-Tek chamber slides were treated with DXR (50 nM) and OLEU $(100 \mu \mathrm{g} / \mathrm{mL}, 200 \mu \mathrm{g} / \mathrm{mL})$, alone and in cotreatment, for $24 \mathrm{~h}$. After a wash with cold phosphatebuffered saline (PBS), they were fixed in methanol for 10 min at $-20^{\circ} \mathrm{C}$, air-dried, washed again with PBS, incubated in $3 \% \mathrm{H}_{2} \mathrm{O}_{2}$ in methanol for $10 \mathrm{~min}$, incubated in $50 \mathrm{mM}$ ammonium chloride in PBS for $15 \mathrm{~min}$ at room 
temperature and then washed with PBS. After blocking non-specific protein binding by incubation with blocking serum (Ultra Vision Detection System Anti-Polyvalent, Lab Vision Corporation, USA) for $20 \mathrm{~min}$ at room temperature and washing with $\mathrm{PBS}$, the cells were incubated with primary antibody (LC3 rabbit polyclonal antibody; Novus, USA) for $60 \mathrm{~min}$ at room temperature. After another wash with cold PBS, the cells were treated with fluorescein isothiocyanate (FITC)-labeled secondary antibody (Santa Cruz Biotechnology, USA) for $60 \mathrm{~min}$ at room temperature, washed again with PBS, mounted with Aqua Poly Mount (Polysciences, Germany) and analyzed using a Nikon fluorescence microscope with a wide-band green filter.

\subsection{Protein Isolation}

After 48h treatment with the appropriate factors cells, grown in $25-\mathrm{cm}^{2}$ culture flasks, were washed twice with ice-cold PBS and scraped into chilled PBS with protease inhibitors. The cells were pelleted and the pellets were resuspended in cell lysis buffer containing Tris- $\mathrm{HCl}, \mathrm{NaCl}$, $\mathrm{NaF}, \mathrm{Na}_{4} \mathrm{P}_{2} \mathrm{O}_{7}, \mathrm{Na}_{3} \mathrm{VO}_{4}$, DTT, Triton $\mathrm{X}-100$, protease inhibitors and phosphatase inhibitor cocktail I (Sigma, USA) 1/100. Cells were passed through a 21 gauge needle, followed by incubation on ice for 20 minutes. After centrifugation the supernatants were collected and assayed for protein concentration using the Bradford method (BioRad Laboratories, USA).

\subsubsection{Western Blot Analysis}

$20 \mu \mathrm{g}$ of each protein sample were resolved on SDS 4$15 \%$ gradient PAGE and electrophoretically transferred to nitrocellulose (Bio-Rad Laboratories, USA) for overnight incubation, at $4^{\circ} \mathrm{C}$, with LC3 rabbit polyclonal antibody (Novus, USA). Blots were then probed with peroxidaseconjugated species appropriate secondary antibodies (Santa Cruz Biotechnology, USA) and visualized by ECL (GE Healthcare, Germany) and exposed to an X-ray film (Kodak, Germany). Equal protein loading was verified using anti-GAPDH monoclonal antibody (Santa Cruz Biotechnology, USA). Intensity ratios of the LC3-II over LC3-I proteins were calculated and the control sample was used as reference value.

\subsection{Cell Extraction and Sample Preparation for NMR Experiments}

Cell samples (approximately $10^{7}$ cells/NMR sample) were extracted by the methanol-chloroform-water (M/C) protocol described by Le Belle et al. [17] ( $\mathrm{n}=5 /$ group). Methanol and chloroform (in reagent-grade) were added to the frozen cell pellets $\left(4^{\circ} \mathrm{C}\right)$ in a ratio of $2: 1(\mathrm{v} / \mathrm{v} ; 250$ $\mu \mathrm{L} /$ cell pellet). The tissue-solvent mixture was allowed to thaw before being transferred to centrifuge tubes. The cell pellet-solvent mixture was sonicated. After approximately $15 \mathrm{~min}$ in contact with the initially added solvents, chloroform and distilled water were added to the samples in a ratio of 1:1 $(250 \mu \mathrm{L} /$ cell pellet $)$, in order to form an emulsion. The samples were then centrifuged at 13,000 rpm for $20 \mathrm{~min}$. The upper phase (methanol and water) was separated from the lower (organic) phase and both fractions were dried at room temperature using a speedvac. The protein pellets from the cells were re-extracted, but the separated fractions were pooled with the original extracted fractions before drying the samples.

The aqueous cell extracts were reconstituted in $700 \mu \mathrm{L}$ of $99.9 \% \mathrm{D}_{2} \mathrm{O}$ at $\mathrm{pH} 7.4$ phosphate buffered (150 mM) containing $0.01 \%$ 1,1,2,2-tetradeutero-3-trimethylsilylpropionic acid (TSP) as internal standard and $0.2 \mathrm{mM} \mathrm{NaN}_{3}$ for microbial growth inhibition. The corresponding lipid cell extracts were reconstituted in $700 \mu \mathrm{L}$ of $\mathrm{CDCl}_{3}$ using tetramethylsilane (TMS) as an internal standard for further NMR experiments.

\subsubsection{NMR Experiments}

All NMR experiments were acquired on a $600 \mathrm{MHz}$ Avance III spectrometer (Bruker GmbH, Germany) equipped with a B-ACS-60 sample changer at temperature probe $27^{\circ} \mathrm{C}$. For each sample $1 \mathrm{D} 1 \mathrm{H}$ NMR (noesypr1d pulse sequence) and ultra-fast $J$-resolved spectra were acquired with water signal suppression during relaxation and mixing time. For the 1D spectra 32 free induction decays (FIDs) were collected into 64k data points at a spectral width of $12335.5 \mathrm{~Hz}$ and with acquisition time of $2.65 \mathrm{~s}$, relaxation delay of $4 \mathrm{~s}$ and mixing time $0.010 \mathrm{~s}$. The NMR spectra were phased corrected and referenced to the chemical shift of TSP at 0.00 ppm using the TOPSPIN v.3.1 software (Bruker BioSpin). The $J$-resolved experiments were acquired with $64 \mathrm{k}$ points for a spectral width of $10000 \mathrm{~Hz}$. Prior to Fourier transformation an exponential weighting factor corresponding to a line broadening of $0.3 \mathrm{~Hz}$ was applied. For selected samples 2D HSQC heteronuclear and 2D COSY and TOCSY homonuclear spectra were recorded to aid resonance assignment according to spectral details described elsewhere [18].

\subsubsection{Data Reduction of the NMR Spectra and Univariate Statistical Analysis}

NMR spectra (spectral width from 9.49 to $0.71 \mathrm{ppm}$ ) were segmented into 431 chemical shift regions of 0.02 ppm width using the software package AMIX Statistics v.3.9.14 (Analysis of MIXtures, Bruker BioSpin) and the integrals were calculated. Regions of a) 7.67-7.70 ppm (unknown peak), b) 4.85-4.70 ppm (residual water suppression trace), b) 3.38-3.34 ppm (methanol) and c) 0.71-0.00 ppm (region with no significant information) were removed from the data set before the application of AMIX package. Data were normalized according to the total intensity of the spectra.

\subsection{Statistical Analysis}

All the variables are expressed as mean \pm standard error of the mean and compared among groups using One-way Analysis of Variance model (ANOVA) with Bonferroni correction and Tukey post-hoc analysis via the software package IBM SPSS Statistics v. 21. A p value $<0.05$ was considered to be statistically significant. For the NMR analysis, the $z$-scores were also calculated from normalized bucket integrals and averaging for each group according to the formula $z$-score $=\left(\operatorname{mean}_{\text {Groupi }}-\right.$ mean $\left._{\text {Control }}\right) / \mathrm{SD}_{\text {control }}$, in order to highlight the metabolites which are responsible for the differentiation of the metabolic profiles between the four groups (doxorubicin, oleuropein, doxorubicinoleuropein, control). 


\section{Results}

\subsection{Cytotoxicity / MTT Assay}

Increasing concentrations of OLEU (3-400 $\mu \mathrm{g} / \mathrm{mL})$ produced a dose-dependent inhibition in the proliferation of PC-3 cells (Figure 1A). Continuous exposure to DXR (3-100 $\mathrm{nM}$ ) produced a maximal inhibition of $66 \%$ on the growth of PC-3 cells at the high doses tested (Figure 1B). The co-treatment of PC-3 cells with DXR (3-100 nM) and $100 \mu \mathrm{g} / \mathrm{mL}$ of OLEU did not alter the cell proliferation inhibition obtained by DXR alone, whereas the cotreatment of PC-3 cells with DXR (3-100 nM) and 200 $\mu \mathrm{g} / \mathrm{mL}$ of OLEU resulted in an additive inhibition of cell proliferation even at very low doses of DXR (3-12,5 nM) (Figure 1B).

A

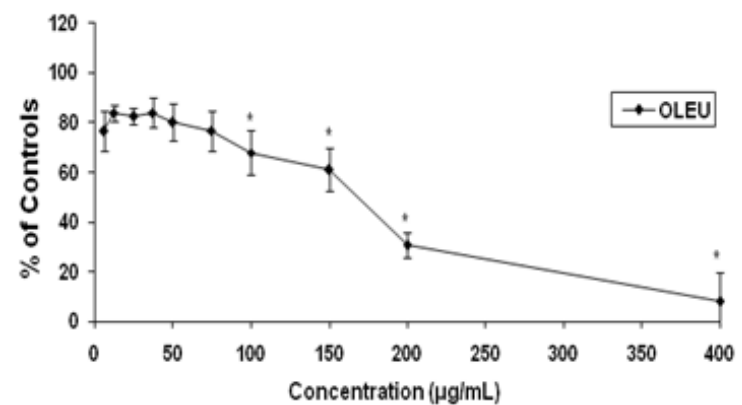

B

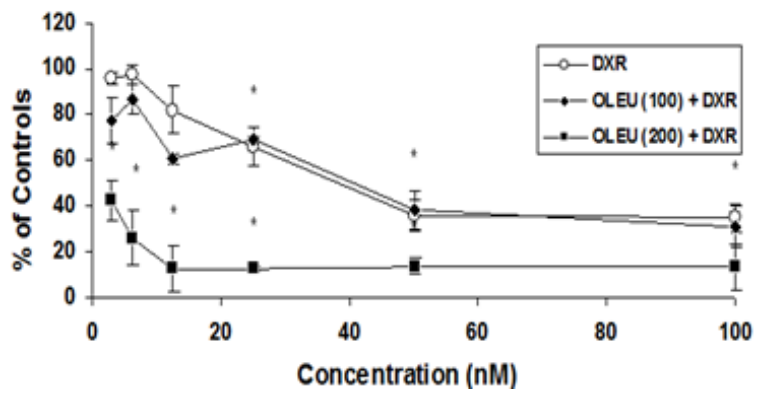

Figure 1. Dose-response curves for the continuous administration (96 hr) of a) OLEU alone (3-400 $\mu \mathrm{g} / \mathrm{mL})$, b) DXR alone (3-100 $\mathrm{nM}$ ) and DXR in co-treatment with either $100 \mu \mathrm{g} / \mathrm{mL}$ OLEU or $200 \mu \mathrm{g} / \mathrm{mL}$ OLEU on PC-3 cells, as assessed by the MTT assay. Results are expressed as percentage of controls. Statistical analysis was performed in triplicate determination at $\mathrm{p}<0.05$

\subsection{Flow Cytometry}

The cell cycle distribution showed that treatment of the PC-3 cells with $200 \mu \mathrm{g} / \mathrm{mL}$ of OLEU for 24,48 and 72 hours produced a marginal increase in the cell distribution in G0/G1 phase, while treatment with $50 \mathrm{nM}$ of DXR showed the expected G2/M phase blockade and suppression of the $\mathrm{S}$ phase. The addition of OLEU to the treatment with DXR did not alter the effect exhibited by DXR alone (Figure 2A, Figure 2B, Figure 2C). Additionally, the combination of Annexin V-FITC and PI staining allows differentiation between early apoptotic cells, late apoptotic cells, necrotic cells and viable cells. The addition of OLEU to DXR, after 48 hours exposure, produced evidence of late apoptosis (ranging from 10$15 \%)$ above that noted by each treatment alone, under these experimental conditions (data not shown).

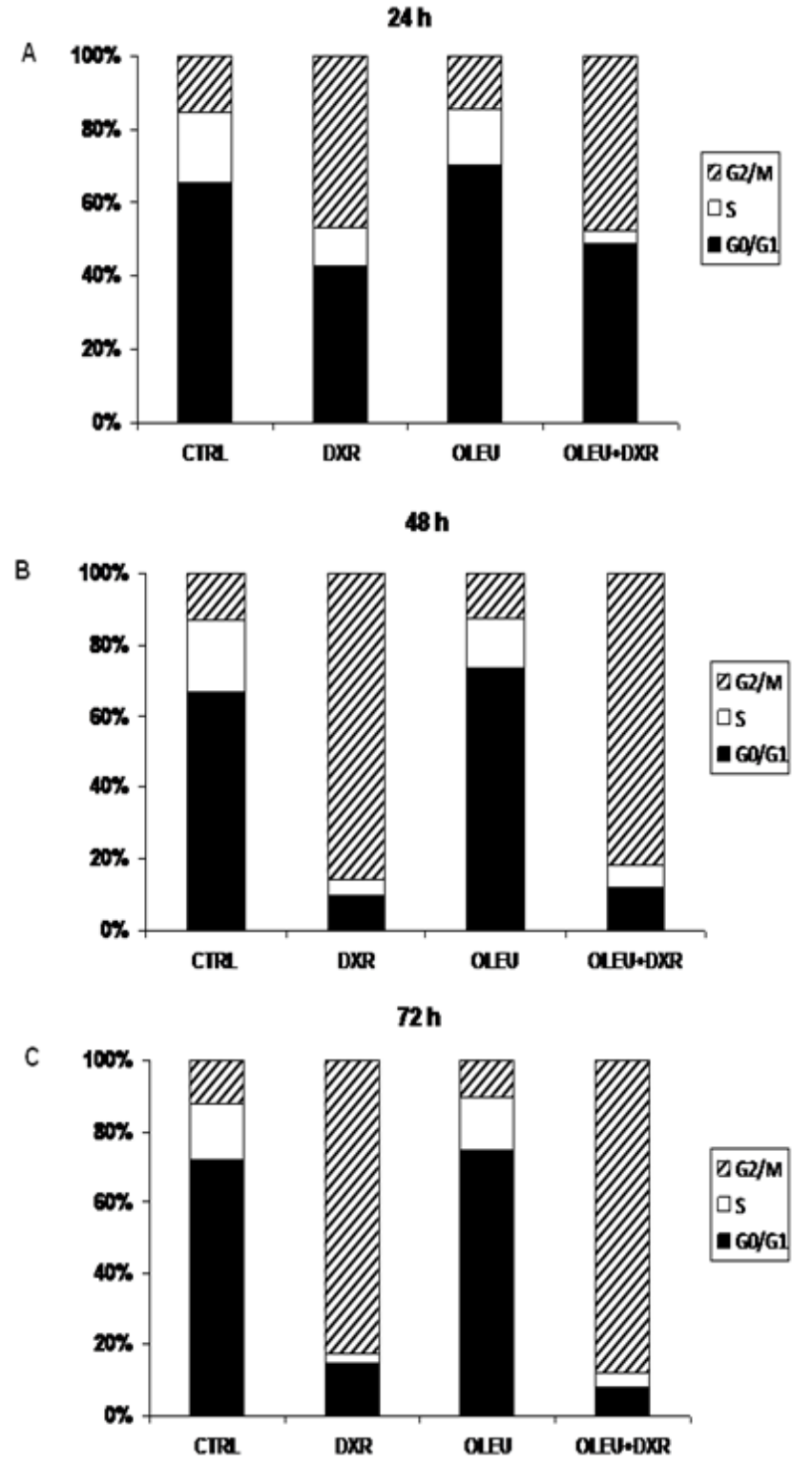

Figure 2. Flow cytometry graphs showing DNA content in PC-3 cells, after a) 24 hours b) 48 hours and c) 72 hours of exposure to DXR (50 nM) and OLEU $(200 \mu \mathrm{g} / \mathrm{mL})$, alone and in co-treatment in comparison to control cells (CTRL). Quantification of G0/G1, S and G2/M phases was based on staining by the DNA binding dye propidium iodide.

\subsection{Autophagy Imaging}

The immunofluorescence experiments revealed the induction of autophagy, in PC-3 cells that received OLEU, DXR and the co-treatment scheme. It was noticed a more pronounced signal in cells that received the combination of $50 \mathrm{nM}$ of DXR with $200 \mu \mathrm{g} / \mathrm{mL}$ of OLEU (Figure 3).

\subsection{Immunoblot Analysis for LC3}

The unconjugated (LC3-I) and conjugated forms (LC3-II) of LC3 in PC-3 cells are resolved by protein electrophoresis as two distinct bands. In fact, treatment with $50 \mathrm{nM}$ DXR, $200 \mu \mathrm{g} / \mathrm{mL}$ OLEU and the co-treatment scheme (DXR + OLEU) resulted in the induction of LC3 membrane translocation, the disappearance of LC3-I protein and the consequent increase in the LC3-II/LC3-I ratio. The enhancement of autophagy is even more evident following the co-treatment of $50 \mathrm{nM}$ DXR and $200 \mu \mathrm{g} / \mathrm{mL}$ OLEU (Figure 4). 
NEGATIVE CONTROL

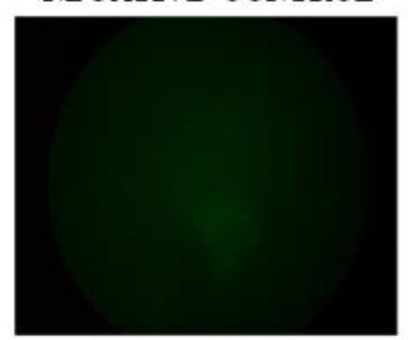

$\operatorname{DXR}(50 \mathrm{nM})$
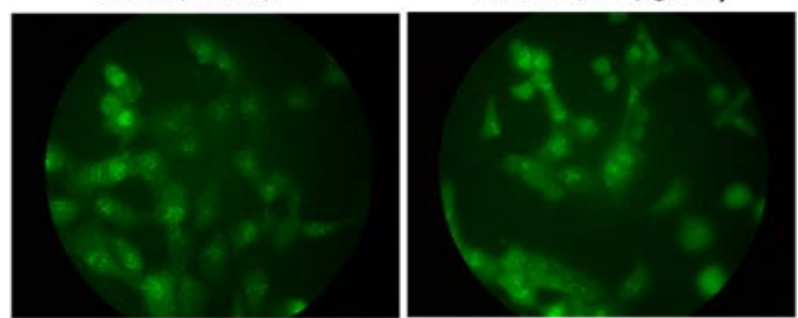

$\operatorname{DXR}(50 \mathrm{nM})+$ OLEU $(200 \mu \mathrm{g} / \mathrm{mL})$



Figure 3. An example of the expression of LC3 (autophagic marker) in PC-3 cells in response to DXR (50 nM) and OLEU (200 $\mu \mathrm{g} / \mathrm{mL})$, alone and in co-treatment, by immunofluerescence, as described in the Materials and Methods section. All treated samples enhanced the expression of LC3 as compared to controls. Note the presence of autophagosomes in treated cells (Magnification: x60)
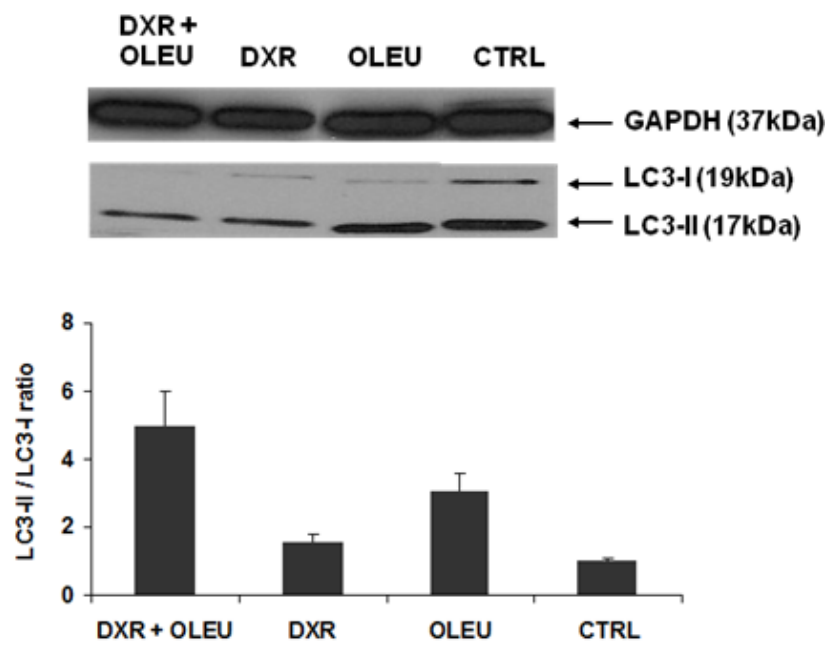

Figure 4. Representative Western blot and densitometry of the expression of the unconjugated (LC3-I) and conjugated forms (LC3-II) of LC3 in PC-3 cells. The upper band (LC3-I) is the autophagy-inactive form of the protein and the lower one (LC3-II) is the active-membrane bound form. The analysis of the intensity of LC3-I and LC3-II by western blot was expressed as a ratio (LC3-II/LC3-I) of the positive control and was normalized against the respective GAPDH signal. DXR (50nM), OLEU (200 $\mu \mathrm{g} / \mathrm{mL})$, DXR (50nM) + OLEU $(200 \mu \mathrm{g} / \mathrm{mL})$

\subsection{Metabolic Profiling}

Differences in the metabolic profile between different groups were revealed using NMR based metabolic profiling. A typical spectrum of cell extracts is illustrated in Figure 5.
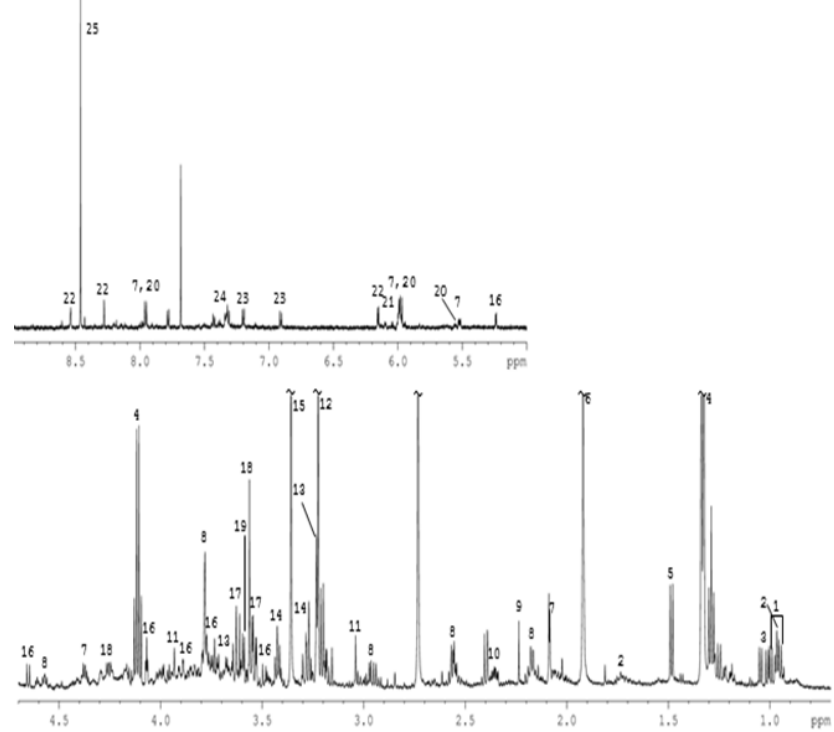

Figure 5. $1 \mathrm{H}$ NMR spectrum of a representative control sample along with the assignment of metabolite signals.

[1: Isoleucine; 2: Leucine; 3: Valine; 4: Lactate; 5: Alanine; 6: Acetate; 7: UDP-N-Acetylglucosamine; 8: Glutathione; 9: Acetone; 10: Glutamate; 11: creatine or creatine phosphate; 12: O-Phosphocholine; 13: sn-Glycero-3-Phosphocholine; 14: Taurine; 15: Methanol; 16: Glucose; 17: Myo-Inositol; 18: Glycine; 19: Threonine; 20: UDP-N-Acetylgalactosamine; 21: NAD+; 22: AXP; 23:Tyrosine; 24: Phenylalanine; 25: Formate]

Twenty-eight metabolites were assigned deviation from controls (according to the normalized data) after different treatments is depicted by z-scores in Table 1.

Table 1. ${ }^{1} \mathrm{H}$ NMR chemical shifts and assignments for cell metabolites and the deviation of each treatment compared to control as depicted by $z$-scores (z-scores are colored as a heat map for easier interpretation). ${ }^{*} p<0.05$ compared to control samples. DXR (50nM), OLEU (200 $\mu \mathrm{g} / \mathrm{mL})$, DXR (50nM) + OLEU (200 $\mu \mathrm{g} / \mathrm{mL})$

\begin{tabular}{|c|c|c|c|c|}
\hline \multirow{2}{*}{ Metabolites } & \multirow{2}{*}{$\delta,{ }^{1} \mathrm{H}(\mathrm{ppm})$} & \multicolumn{3}{|c|}{$z$-scores $v$ s control } \\
\cline { 3 - 5 } & & $\mathrm{DXR}$ & $\mathrm{DXR}+$ OLEU & OLEU \\
\hline Formate & $8.47-8.45$ & -0.01 & 0.71 & 0.09 \\
\hline Phenylalanine & $7.45-7.43$ & -2.87 & 0.60 & 0.05 \\
\hline Tyrosine & $7.21-7.19$ & -1.49 & 1.18 & $-4.69^{*}$ \\
\hline ATP & $6.15-6.13$ & -0.86 & $15.15^{*}$ & -5.27 \\
\hline NAD & 6.05 & -0.06 & -0.06 & -1.18 \\
\hline $\begin{array}{c}\text { UDP-N- } \\
\text { Acetylglucosamine }\end{array}$ & $5.53-5.51$ & 1.40 & $14.50^{*}$ & -0.76 \\
\hline Glucose & 5.23 & 1.12 & $5.83^{*}$ & 0.91 \\
\hline Myo-Inositol & 4.07 & -0.11 & 0.02 & 0.35 \\
\hline Glycine & 3.57 & -0.55 & 1.13 & 1.11 \\
\hline Taurine & 3.43 & $-2.71^{*}$ & -1.01 & -0.79 \\
\hline $\begin{array}{c}\text { sn-Glycero- } \\
\text { Phosphocholine }\end{array}$ & 3.23 & -1.56 & -0.14 & 2.03 \\
\hline Creatine & $3.05-3.03$ & -1.45 & 0.05 & 1.56 \\
\hline Glutathione & 2.55 & -2.50 & $-4.59^{*}$ & $-4.10^{*}$ \\
\hline Succinate & 2.41 & 1.04 & 0.03 & 0.00 \\
\hline Glutamate & 2.35 & -0.58 & -0.90 & -0.95 \\
\hline Acetate & $1.93-1.91$ & -2.95 & -4.03 & -4.61 \\
\hline Alanine & 1.49 & -0.27 & -1.71 & -1.71 \\
\hline Lactate & 1.33 & $0.61^{*}$ & -0.30 & -1.11 \\
\hline $\begin{array}{c}\text { 3-Hydroxy- } \\
\text { isovalerate }\end{array}$ & 1.25 & 5.32 & 0.43 & 2.64 \\
\hline Valine & 1.05 & 0.13 & 0.41 & -0.65 \\
\hline Leucine & 0.97 & -0.64 & -0.24 & -0.76 \\
\hline Isoleucine & 0.95 & 0.02 & 0.55 & -0.21 \\
\hline & & & & \\
\hline
\end{tabular}


Univariate ANOVA statistical analysis results are incorporated also in the table as indicated with the asterisk when statistical significant difference is observed. Significant alterations in DXR treatment are phenylalanine, taurine and acetate exhibiting a downregulation, while 3Hydroxy-isovalerate exhibits an interesting upregulation primarily in DXR and secondary in OLEU treatment. Tyrosine, ATP and acetate exhibit a strong reduction for the OLEU compared to control group. More importantly substantial increase of ATP, UDP-N-Acetylglucosamine and glucose levels is observed in DXR + OLEU cotreatment as compared to control. Furthermore a concomitant reduction for glutathione and acetate is shown in all treated cell samples.

\section{Discussion}

To overcome advanced prostate cancer, the commonly used treatment to date is the inhibition of androgen production and/or androgen function. In the past the development of anticancer drugs has traditionally relied on in vitro tests aimed almost exclusively at assessing the potential of direct killing or growth inhibiting of cancer cell lines. However, accumulating evidence indicates that the in vivo response to anticancer therapies is mostly influenced by the toxicity and side-effects that the chemotherapeutic schemes may confer. Doxorubicin, a known topoisomerase II inhibitor, producing G2/M arrest and apoptosis in a cellular level, affects significantly the growth of prostate cancer cells. However, the severe cardiotoxicity that gradually develops, erases many questions about the "'pour ou contre' of DXR applications. Therefore, many researchers have launched studies aiming at reducing the DXR-induced toxicity $[19,20,21]$. These studies have until now, focused on the induction of apoptosis, while the present study addresses the combination of DXR with oleuropein, a natural product, in the field of prostate cancer, with regard to the more novel mechanism of autophagy.

Nowadays, there is a strong evidence for the protective role of oleuropein against many types of cancer [22,23,24]. More specifically, Acquaviva et al, have recently demonstrated the antiproliferative effect of oleuropein in the androgen sensitive LNCaP and in the androgen insensitive DU145 prostate cancer cell line which are characterized by low and moderate tumorigenicity, respectively [25]. Herein, it is studied the antiproliferative effect of oleuropein and its potent synergistic action with $\mathrm{DXR}$, in the androgen insensitive p53-negative human PC-3 prostate cancer cell line, originally derived from the bone metastatic lesion of a prostate cancer patient. Because the characteristics of PC-3 cells consent with many of the clinical aspects of the hormone refractory prostatic cancer, this cell line has been widely used as a model of prostate cancer progression [15].

This study demonstrates that both DXR and oleuropein inhibit PC-3 cells proliferation in a dose-dependent manner, while the co-treatment with DXR and oleuropein results in an additive inhibition of cell proliferation even at very low doses of DXR. This finding is considered of great importance, as oleuropein is capable of lowering significantly the cytotoxic dose of DXR, while obtaining an outstanding antiproliferative effect in prostate cancer cells. Additionally, the fact that the effectiveness of DXR in inhibiting cell proliferation is not compromised when oleuropein is present, which may not always be taken for granted, demonstrates that the anticancer activity of DXR is not alleviated by oleuropein.

Furthermore, the fact that there was no significant alteration of the cell cycle distribution and that there was a marginal increase on the rate of late apoptosis, between the cell samples treated with the combination scheme and the treatment solely with DXR, led the authors to investigate the implication of another intracellular molecular mechanism, autophagy. Indeed, the results show that DXR and OLEU produce a remarkable induction of autophagy, which is more pronounced following the co-treatment scheme. This finding was confirmed by both immunofluorescence and immunoblotting. Although autophagy has been proposed as a cell death process, the role of autophagy in cancer cell death is still in dispute. Previous studies have shown that when tumor cells are deprived of growth/survival factors, autophagy is increased to prevent the cells from dying [26]. In addition, when autophagy is prevented under these conditions, the cells undergo apoptosis. Therefore, autophagy seems to play a role in preventing cellular apoptosis from nutritional stress in cancer cells. In contrast, several other studies have demonstrated that autophagy has an anti-cancer effect. However, it is unclear whether autophagy is the mechanism for cell death or a reactive mechanism by which the cell is trying to survive the chemotherapy [27].

Cancer cells often modify their metabolism by promoting diverse biosynthetic pathways to adapt to the environment and to ensure their rapid proliferation. Hence, a growing appreciation of the role of autophagy in controlling cellular metabolism has fuelled immense interest in elucidating how dysfunctional autophagy influences metabolic disorders and metabolic adaptation in diseases such as cancer. Furthermore, autophagy can mobilize diverse cellular energy stores to replenish metabolites during both normal and stressed conditions. Recent studies delineate a direct crosstalk between metabolic enzymes and autophagy and that, as an intracellular recycling system, autophagy is highly important for cell metabolism and nutrient allocation. Therefore, it is highly supported that there is a direct regulation of autophagy by the provided cell metabolic context [28]. In the present study, treated cell samples exhibited significant metabolite alterations, which highly correlate with the cell autophagic response. Among the identified metabolites, those involved in energy production (ATP, glucose and glutamate), amino acid metabolism (glycine, alanine, valine, leucine and tyrosine), as well as in oxidative profile (glutathione), of rapidly dividing tumor cells seemed to be starring in separating the groups DXR, OLEU, DXR+OLEU and Control. These results indicate that several specific metabolic pathways are disturbed in prostate cancer tissue. Sustained autophagy under conditions of protracted starvation has been proposed to lead to cell death; thus, the survival or death consequences of autophagy are condition-dependent.

Growth in a hostile environment, inefficient utilization of glucose and defective autophagy predict that prostate cancers may be particularly sensitive to therapies that inflict metabolic stress. It is, therefore, hypothesized that 
prostate cancer is metabolically fragile because of dependence on glycolysis and impaired autophagy [10]. Specifically, among the treated cells, the downregulation of taurine and glutamate, characteristics of protein catabolism taking place in autophagy, correlate well with the proposed mechanism of action of the treatments tested. Moreover, the concominent downregulation of reduced glutathione not only highlights the antioxidant role of DXR and the even stronger antioxidant effect of OLEU, but also confirms the high endogenous glutathione levels of cancer cells as a result of the cancer-mediated cell stress [29]. It is also noteworthy, that human prostate cancer cell lines of varying degrees of aggressive behavior have distinct redox properties and that each cell line shows distinct cytotoxic responses to low-molecularweight redox-modulating compounds [30].

An observation of outmost interest in the metabolic profiling analysis is the outstanding increase of UDP$\mathrm{N}$-Acetylglucosamine and ATP along with glucose in DXR and OLEU co-treatment compared to controls. Although ATP is mostly used to provide energy for cellular processes, it also directly links energy metabolism to signaling. Indeed, Kawaguchi et al. recently demonstrated that cancer cells promote autophagy to overcome the energy shortage from glycolysis, the major energy source, by maintaining mitochondrial activity to produce ATP necessary for survival [31].These data show that mitochondria are not simply the power plants of cells generating ATP, but are closely related to several types of cell death and autophagy. Uridine diphosphate $\mathrm{N}$-acetylglucosamine (UDP-GlcNAc) on the other hand, which is substantially regulated by the combination scheme (DXR+OLEU) as well, is the high energy donor substrate for O-linked $\mathrm{N}$-acetylglucosamine. UDP-GlcNAc sits at the nexus of glucose, nitrogen, fatty acid and nucleic acid metabolic pathways, all of which dynamically influence its cellular concentration. Mammals respond to nutrient excess and different other forms of stress (heat shock, oxidative, osmotic, ER, glucose) by addition of O-linked $\mathrm{N}$-acetylglucosamine at serine and threonine residues of nucleocytoplasmic proteins, which is directly associated with autophagy and with regulation of endoplasmic reticulum stress and cell viability [32,33]. Glycosylation can alter protein function and has a key role in many important biological processes incancerincluding cell adhesion, migration, interactions with the cell matrix, immune surveillance, cell signaling and cellular metabolism; altered glycosylation inprostate cancer in particular, might modify some or all of these processes[34,35,36]. More specifically, Itkonen et al., showed that UDP-GlcNAc is activated in PC-3 prostate cancer cells and can act as a modulator of prostate cancer growth, which reinforces the notion that these often overlooked protein modifications have the potential to improve risk stratification and therapeutic strategies in patients with prostate cancer [37].

Finally, our results agree with those, recently reported, suggesting the beneficial effects of oleuropein against cancer, ageing, neurodegeneration, diabetes and diseases implying autophagy dysfunction [38,39]. Further understanding of the molecular mechanisms which are activated in response to different stimuli is therefore important towards the pharmaceutical development of novel treatment methods for prostate cancer.

Concluding remarks: The results of the present study provide provocative evidence that oleuropein is a powerful sensitizer of doxorubicin-induced killing of prostate cancer. Because it is a bioactive natural product which is anyway obtained by food consumption, it is regarded as a very promising alternative, not only for the lack of toxicity when administered in a co-treatment scheme with doxorubicin, but also for providing alleviation of the side-effects of DXR in cancer patients.

\section{References}

[1] Keys A, A.C., Van Buchem FSP The diet and all-causes death rate in the Seven Countries Study. Lancet, 1981. 2(8237): p. 58-61.

[2] Boring, C.C., T.S. Squires, and T. Tong, Cancer statistics, 1992. CA Cancer J Clin, 1992. 42(1): p. 19-38.

[3] Minotti, G., et al., Anthracyclines: molecular advances and pharmacologic developments in antitumor activity and cardiotoxicity. Pharmacol Rev, 2004. 56(2): p. 185-229.

[4] Owen, R.W., et al., Olive-oil consumption and health: the possible role of antioxidants. Lancet Oncol, 2000. 1: p. 107-12.

[5] Tripoli, E., et al., The phenolic compounds of olive oil: structure, biological activity and beneficial effects on human health. Nutr Res Rev, 2005. 18(1): p. 98-112.

[6] Goldsmith, C.D., et al., Phytochemical properties and antiproliferative activity of Olea europaea L. leaf extracts against pancreatic cancer cells. Molecules, 2015. 20(7): p. 12992-3004.

[7] Fayyaz, S., et al., Oleuropein mediated targeting of signaling network in cancer. Curr Top Med Chem, 2016. 16.

[8] Andreadou, I., et al., The olive constituent oleuropein exhibits anti-ischemic, antioxidative, and hypolipidemic effects in anesthetized rabbits. J Nutr, 2006. 136(8): p. 2213-9.

[9] Andreadou, I., et al., Acute doxorubicin cardiotoxicity is successfully treated with the phytochemical oleuropein through suppression of oxidative and nitrosative stress. J Mol Cell Cardiol, 2007. 42(3): p. 549-58.

[10] DiPaola, R.S., et al., Therapeutic starvation and autophagy in prostate cancer: a new paradigm for targeting metabolism in cancer therapy. Prostate, 2008. 68(16): p. 1743-52.

[11] Mathew, R., V. Karantza-Wadsworth, and E. White, Role of autophagy in cancer. Nat Rev Cancer, 2007. 7(12): p. 961-7.

[12] Shintani, T. and D.J. Klionsky, Autophagy in health and disease: a double-edged sword. Science, 2004. 306(5698): p. 990-5.

[13] Kabeya, Y., et al., LC3, a mammalian homologue of yeast Apg8p, is localized in autophagosome membranes after processing. EMBO J, 2000. 19(21): p. 5720-8.

[14] Zoidou, E., et al., Identification of Throuba Thassos, a traditional Greek table olive variety, as a nutritional rich source of oleuropein. J Agric Food Chem, 2010. 58(1): p. 46-50.

[15] Kaighn, M.E., et al., Establishment and characterization of a human prostatic carcinoma cell line (PC-3). Invest Urol, 1979. 17(1): p. 16-23.

[16] Vistica, D.T., et al., Tetrazolium-based assays for cellular viability: a critical examination of selected parameters affecting formazan production. Cancer Res, 1991. 51(10): p. 2515-20.

[17] Le Belle, J.E., et al., A comparison of cell and tissue extraction techniques using high-resolution 1H-NMR spectroscopy. NMR Biomed, 2002. 15(1): p. 37-44.

[18] Pechlivanis, A., et al., (1)H NMR-based metabonomic investigation of the effect of two different exercise sessions on the metabolic fingerprint of human urine. J Proteome Res, 2010. 9(12): p. 6405-16.

[19] Tenta, R., et al., Bone microenvironment-related growth factors modulate differentially the anticancer actions of zoledronic acid and doxorubicin on PC-3 prostate cancer cells. Prostate, 2004. 59(2): p. 120-31.

[20] Kapadia, G.J., et al., Synergistic cytotoxicity of red beetroot (Beta vulgaris L.) extract with doxorubicin in human pancreatic, breast and prostate cancer cell lines. J Complement Integr Med, 2013. 10 p. 113-122. 
[21] Das, A., et al., Sildenafil increases chemotherapeutic efficacy of doxorubicin in prostate cancer and ameliorates cardiac dysfunction. Proc Natl Acad Sci U S A, 2010. 107(42): p. 18202-7.

[22] Katsoulieris, E.N., The olive leaf extract oleuropein exerts protective effects against oxidant-induced cell death, concurrently displaying pro-oxidant activity in human hepatocarcinoma cells. Redox Rep, 2016. 21(2): p. 90-7.

[23] Giner, E., et al., Chemopreventive effect of oleuropein in colitis associated colorectal cancer in c57bl/6 mice. Mol Nutr Food Res, 2016. 60(2): p. 242-55

[24] Cheng, J.S., et al., The effect of oleuropein from olive leaf (Olea europaea) extract on $\mathrm{Ca}(2+)$ homeostasis, cytotoxicity, cell cycle distribution and ROS signaling in HepG2 human hepatoma cells. Food Chem Toxicol, 2016. 91: p. 151-66.

[25] Acquaviva, R., et al., Antiproliferative effect of oleuropein in prostate cell lines. Int J Oncol, 2012. 41(1): p. 31-8.

[26] Hippert, M.M., P.S. O'Toole, and A. Thorburn, Autophagy in cancer: good, bad, or both? Cancer Res, 2006. 66(19): p. 9349-51.

[27] Singletary, K. and J. Milner, Diet, autophagy, and cancer: a review. Cancer Epidemiol Biomarkers Prev, 2008. 17(7): p. 1596-610.

[28] Kaur, J. and J. Debnath, Autophagy at the crossroads of catabolism and anabolism. Nat Rev Mol Cell Biol, 2015. 16(8): p. 461-72.

[29] Ortega, A.L., S. Mena, and J.M. Estrela, Glutathione in cancer cell death. Cancers (Basel), 2011. 3(1): p. 1285-310.

[30] Jorgenson, T.C., W. Zhong, and T.D. Oberley, Redox imbalance and biochemical changes in cancer. Cancer Res, 2013. 73(20): p. 6118-23.
[31] Kawaguchi, M., et al., Autophagy is an important metabolic pathway to determine leukemia cell survival following suppression of the glycolytic pathway. Biochem Biophys Res Commun, 2016. 474(1): p. 188-92.

[32] Lilienbaum, A., Relationship between the proteasomal system and autophagy. Int J Biochem Mol Biol, 2013. 4(1): p. 1-26.

[33] Ding, W.X., et al., Linking of autophagy to ubiquitin-proteasome system is important for the regulation of endoplasmic reticulum stress and cell viability. Am J Pathol, 2007. 171(2): p. 513-24.

[34] Copeland, R.J., J.W. Bullen, and G.W. Hart, Cross-talk between GlcNAcylation and phosphorylation: roles in insulin resistance and glucose toxicity. Am J Physiol Endocrinol Metab, 2008. 295(1): p. E17-28.

[35] de Queiroz, R.M., E. Carvalho, and W.B. Dias, O-GlcNAcylation: The Sweet Side of the Cancer. Front Oncol, 2014. 4: p. 132.

[36] Munkley, J., I.G. Mills, and D.J. Elliott, The role of glycans in the development and progression of prostate cancer. Nat Rev Urol, 2016. 13(6): p. 324-33.

[37] Itkonen, H.M., et al., O-GlcNAc transferase integrates metabolic pathways to regulate the stability of c-MYC in human prostate cancer cells. Cancer Res, 2013. 73(16): p. 5277-87.

[38] Leri, M., et al., The polyphenol Oleuropein aglycone hinders the growth of toxic transthyretin amyloid assemblies. J Nutr Biochem, 2016. 30: p. 153-66.

[39] Potocnjak, I., et al., Oral administration of oleuropein attenuates cisplatin-induced acute renal injury in mice through inhibition of ERK signaling. Mol Nutr Food Res, 2016. 60(3): p. 530-41. 